\title{
MORPHOLOGY, BAND STRUCTURE AND OPTICAL PROPERTIES OF ARTIFICIAL OPALS
}

\author{
Davide Comoretto $^{*}$, Eva Pavarini ${ }^{\mathrm{b}}$, Matteo Galli ${ }^{\mathrm{b}}$, Cesare Soci ${ }^{\mathrm{b}}$, Franco Marabelli ${ }^{\mathrm{b}}$, and Lucio \\ Claudio Andreani ${ }^{\mathrm{b}}$ \\ ${ }^{a}$ INFM-INSTM-Dipartimento di Chimica e Chimica Industriale, Università di Genova, via \\ Dodecaneso 31, 20146-Genova, Italy \\ b INFM-Dipartimento di Fisica “A. Volta”, Università di Pavia, via Bassi 7, 27100-Pavia, Italy
}

Bulk polystyrene opals have been grown. Variable incidence angle reflectance spectroscopy is used to probe their photonic band structures. Several different structures are observed and accounted for by theoretical calculations of photonic bands and density of states. The results yield a clear distinction between diffraction in the direction of propagation by the (111) family planes (leading to the formation of the stop band) and diffraction in other directions by higher-order planes (corresponding to excitation of photonic modes in the crystal).

Keywords: photonic crystals, artificial opals

\section{INTRODUCTION}

The optical properties of colloidal photonic crystals have been widely investigated both from the experimental $[1,2,3$, 4] and theoretical [5, 6] point of view. Experimental investigations concerned the properties of the lowest-order gap, such as the attenuation studied by normal-incidence transmission and the evolution of the stop-band in reflectance/transmittance spectra upon varying angles of incidence. Moreover, the role of disorder in Bragg scattering and gap formation has been addressed with measurements on multiple-domain and single-domain crystals. On the theoretical side, the formation of a complete band gap in the inverse opal structure and its fragility against disorder are now well understood. The interplay between photonic bands and reflection/transmission properties has been addressed both with calculations of optical spectra and with symmetry analysis. More recent works focus on the effects of stacking faults on the optical properties.

On the other hand, the assignment of opals optical spectra in the high energy spectral region and for large incidence angles is still debated $[2-4,6]$ since spectral features cannot be unambiguously associated with the presence of a photonic gap [7, 8] and/or with diffraction [9]. Angle-resolved reflectance measurements give evidence of multiple diffraction peaks with small splittings which have been interpreted with concepts related to an anticrossing of photonic levels close to the $\mathrm{U}$ point of the Brollouin zone. However, since reflectance spectra are not only affected by the band gaps but also by the excitation of photonic modes which can be observed only in the density of states, more refined investigations may be suitable to improve the comprehension of the problem. To this end, we report on the optical reflectance at large incidence angles and in a wide spectral region along different crystallographic directions in polystyrene artificial opals. The data are interpreted on the basis of photonic bands and density of states calculations.

\section{EXPERIMENTAL METHODOLOGY}

Bulk polystyrene opals have been grown by monodisperse sphere suspensions in water (Duke Scientific, diameters $222,260,300$ and $426 \mathrm{~nm}$, refractive index 1.59). Small vessels (diameter ranging from 1 to 5 centimeters) are filled with the sphere suspension and placed in a wet environment to reduce the evaporation rate. Opals have a meniscus shape and a glossy surface. When observed at near normal incidence, they show bright and intense reflections (green for 222 and golden for $260 \mathrm{~nm}$ ) while for higher incidence angles, different pale colors appear.

The surface morphology of bulk opal is composed of large domains (hundreds of microns squared) separated by cracks or stacking faults. Domains show triangular packing of the spheres, but with different quality. In disordered domains, several sphere vacancies or dislocations are present. In ordered domains few defects are observed. Figure 1 shows a Scanning Electron Microscopy (SEM) image of the opal $(222 \mathrm{~nm}$ ) surface recorded with a Leo Stereoscan 440 (LEO

*comorett@ chimica.unige.it; phone +39-010-3538736; fax +39-0103536199. 
Electron Microscopy Ltd) electronic microscope. Left panel shows a large area view (about 56x80 $\mu \mathrm{m}^{2}$ ) while in right panel a detail of this picture (about $14 \times 20 \mu \mathrm{m}^{2}$ ) is reported. The surface is very flat on a large scale and shows a regular disposition of the spheres in a triangular lattice as expected for their three-dimensional packing in a facecentered-cubic (fcc) lattice when observed along its (111) direction. This image shows the average surface quality for different samples used in this work. However, for the top quality opals ( $222 \mathrm{~nm}$, see below), better surfaces can be observed. Optical data recorded on the latter will be used for the comparison with theoretical results. The morphology here reported is very different from that of opal films previously investigated, which were characterized by domains of spheres of a few square microns areas showing both triangular and square lattices, tilted with respect to each other and separated by disordered zones or deep dislocations [10, 11]. For the present bulk opal samples, no square lattices are observed and the surface appears as a large triangular lattice of spheres with only a few defects like sphere vacancies and dislocations.
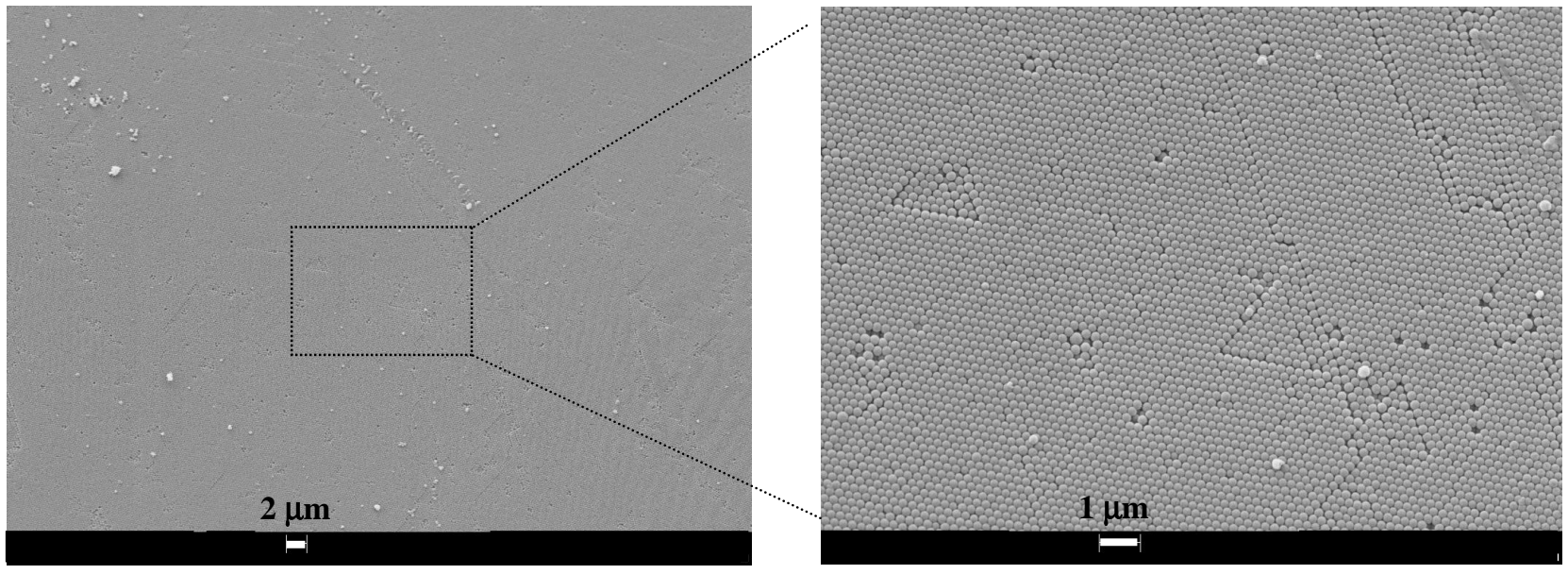

Fig. 1. SEM images of the surface of a $222 \mathrm{~nm}$ opal. Left panel $56 \times 80 \mu \mathrm{m}^{2}$, right panel $14 \times 20 \mu \mathrm{m}^{2}$. Right panel shows an expanded view of the area inside the dashed rectangle. The triangular packing of spheres together with a few dislocation lines and local crystallographic defects.

Unpolarized variable-angle specular reflectance has been measured in the $0.4-4 \mathrm{eV}$ spectral range by means of a Fourier-Transform spectrophotometer Bruker IFS66 with $8 \mathrm{~cm}^{-1}$ spectral resolution. The light of a Xe arc-lamp was collimated and then focused to a spot of 50-100 $\mu \mathrm{m}$ diameter on the sample surface. The sample was placed on a homemade $\theta-2 \theta$ goniometer, that allows the incidence angle to be varied between 5 and 80 degrees, with an angular resolution of $1^{\circ}$ determined by the divergence of the incident light cone. Sample visualization and precise allocation of the light spot on the surface was achieved by an optical microscope mounted on the goniometer. An Ag mirror is used as a reference. The overall error bar of the experimental set-up on the absolute value of the reflectance is within $2 \%$. Particular care has been taken in the alignment of the sample. Since the top quality surface of the investigated sample is located close to one of its sharp border, we used the latter as a macroscopic reference to identify the crystallographic axes of the surface. As a matter of fact, atomic force microscope images recorded at different magnifications showed that the LW crystallographic direction has a well defined offset angle with respect to the sharp border. Therefore the sample was mounted on the micro-reflectometer by aligning the incidence plane with the sharp border and than rotating it by the offset angle. Fig. 2 (left) shows the experimental configuration for reflectance measurements. The incidence angle $\theta$ is measured from the normal to the surface, while $\phi$ (the azimuth angle) is measured from the L-W direction, which is parallel to sphere rows. The set of reflectance spectra recorded with the incidence plane aligned with the L-W direction are six-fold degenerate upon sample rotation (by $\phi$ ) around its normal. Upon rotation of $30^{\circ}$ from $\mathrm{L}-\mathrm{W}$, a different set of reflectance spectra is observed. They still are six-fold degenerate. This fact is puzzling since the $\mathrm{K}$ and $\mathrm{U}$ points of the Brillouin zone (Fig. 2 right) are not identical and two new sets of three-fold degenerate spectra would be expected [12]. 


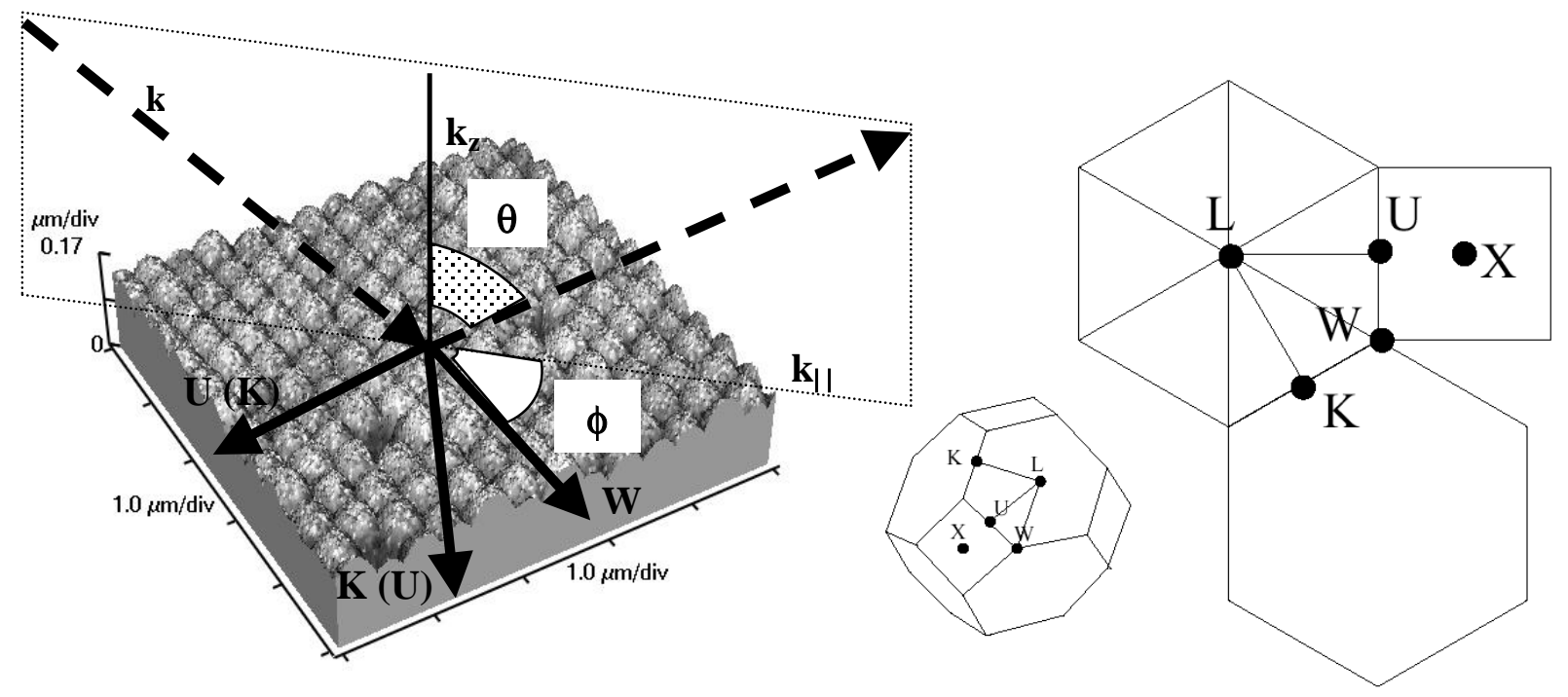

Fig. 2. Left: Scheme of the experimental condition and alignment with respect to the opal $(1,1,1)$ surface (AFM image). Solid lines on the opal surface indicate the intersection with planes containing $\Gamma-\mathrm{W}, \Gamma-\mathrm{K}$ and $\Gamma-\mathrm{U}$ directions; dashed lines on the incidence plane show the wavevector of the incident and reflected beam, with the components $\mathrm{k}_{\|}$(lying on the crystal surface) and $\mathrm{k}_{\mathrm{z}}$ (perpendicular to the crystal surface and parallel to the $\Gamma-\mathrm{L}$ direction). Right: Symmetry points in the Brillouin zone for a fcc lattice.

\section{THEORETICAL MODEL}

In order to calculate the photonic bands we adopt the plane-wave expansion technique to transform the second-order wave equation for the magnetic field into an eigenvalue problem, and calculate the inverse dielectric constant by using the procedure reported in ref. [13] $\left(\varepsilon_{1}=2.53\right.$ (spheres) and $\varepsilon_{2}=1$ (voids), i.e., polystyrene spheres in air). The photonic bands do not exhibit any complete gap in the Brillouin zone (BZ). However, partial gaps do appear along some symmetry lines of the BZ. Among these, the lowest energy gap opens along $\Gamma-\mathrm{L}$ around $\omega \mathrm{a} /(2 \pi \mathrm{c}) \sim 0.6[14]$.

Incomplete gaps behave as stop bands in reflectance spectra when they occur in the direction of propagation. Photonic modes along the propagation direction also contribute to the optical properties and may give rise to additional features in reflectance. In order to distinguish reflectance maxima due to stop-bands from features, which can instead be associated to photonic modes, we first need to calculate the photonic band structure and the density of states (DOS) along the direction of propagation, i.e., for a specified value of the angle of incidence. In the following we will refer to these bands and DOS as reduced bands and reduced DOS, respectively.

The surface of a fcc colloidal crystal is known to be a (111) crystallographic plane, therefore angle-resolved reflectivity experiments are usually performed as a function of the angle of incidence with respect to a (111) surface. We adopt the same convention in our calculations and define a reference frame as follows: $z$ is the $[1,1,1](\Gamma-\mathrm{L})$ direction, $x$ is the $[1,0,-1]$ (a L-W) direction, and $y$ is the $[1,-2,1](\mathrm{L}-\mathrm{K})$ direction. We can therefore express the momentum $\mathbf{k}$ of an incident photon with energy $\hbar \omega$ as follows:

$$
\mathbf{k}=\frac{\omega}{\mathrm{c}}(\sin \theta \cos \varphi, \sin \theta \sin \varphi, \cos \theta)
$$

With our choice of axes, $\theta=0^{\circ}$ corresponds to normal incidence ( $\Gamma$-L direction) and the azimuthal angle $\phi$ is measured from the L-W direction.

The reduced bands and DOS are obtained from the full band structure by using conservation law for both parallel component of momentum modulo a reciprocal lattice vector 


$$
\mathbf{k}_{\|}+\mathbf{G}_{\|}=\frac{\omega}{\mathrm{c}}(\sin \theta \cos \varphi, \sin \theta \sin \varphi, 0)
$$

and energy

$$
\varepsilon_{\mathrm{n}}\left(\mathbf{k}_{\|}, \mathrm{k}_{\mathrm{z}}\right)=\hbar \omega
$$

where $\mathbf{k}_{\|}$is the component of $\mathbf{k}$ parallel to the (111) surface, $\mathbf{G}_{\|}$is the parallel component of a reciprocal lattice vector $\mathbf{G}, \mathrm{k}_{\mathrm{z}}$ is the component of $\mathbf{k}$ parallel to $\Gamma-\mathrm{L}$, and $\varepsilon_{\mathrm{n}}\left(\mathbf{k}_{\|}, \mathrm{k}_{\mathrm{z}}\right)$ is the $\mathrm{n}$-th energy band of a photon with momentum $\mathbf{k}$.
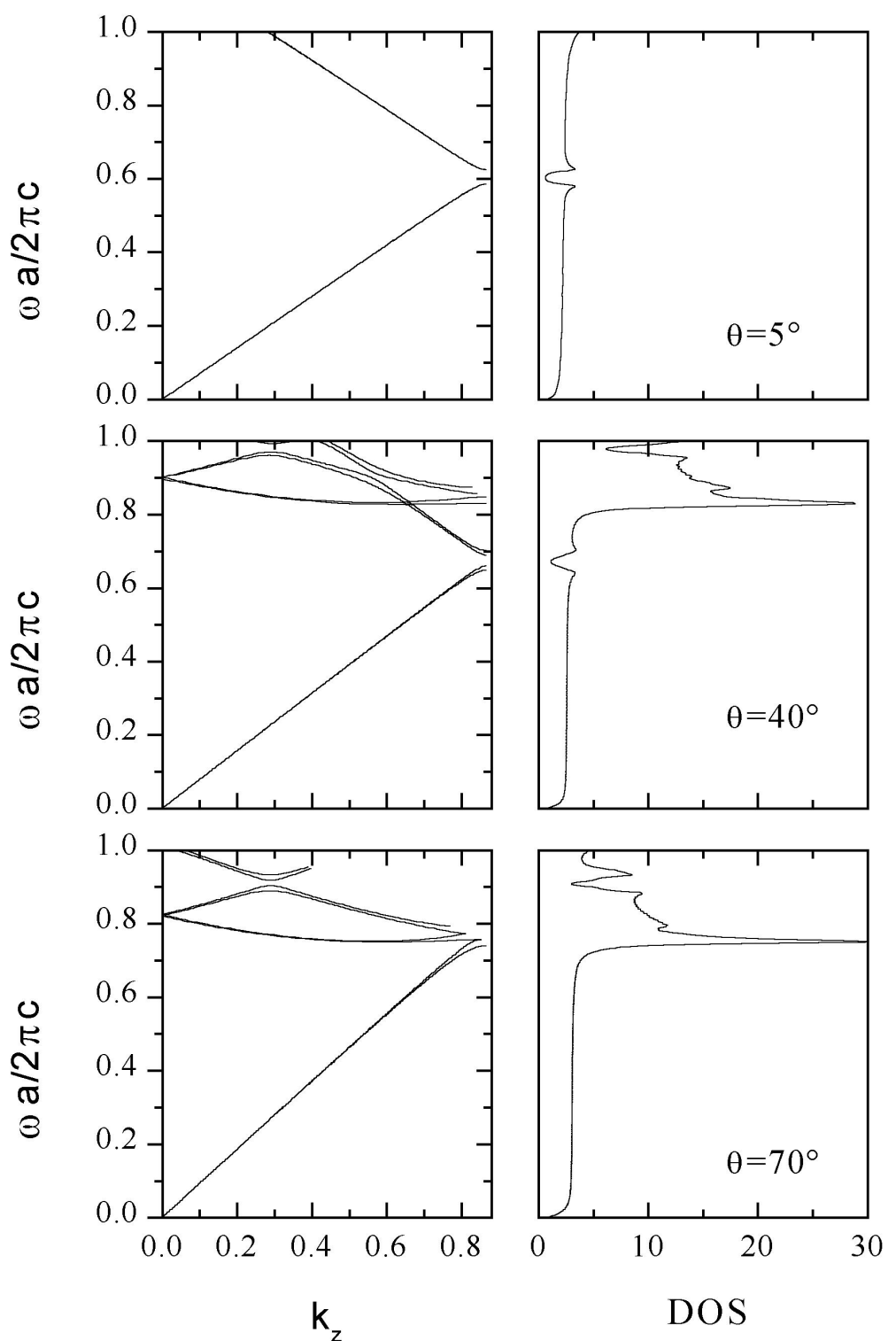

Fig. 3. Reduced photonic band structure as a function of the wavevector component perpendicular to the surface and reduced density of states (DOS) along the L-W direction for different incidence angles. DOS shape highlights either band-gap structures (deeps) as well as singularity points where diffraction in multiple directions takes place. 
We point out that since $\mathbf{k}_{\|}$is fixed by the experimental configuration, the reduced bands depends only on $\mathrm{k}_{\mathrm{z}}$. Reduced bands and DOS are obtained solving system of equations (1), (2) and (3) by a self-consistent procedure.

The reduced DOS obtained from the reduced bands is a one-dimensional density of states, because it is summed only over $\mathrm{k}_{\mathrm{z}}$. An example of the DOS obtained in this way (convoluted with a Lorentzian curve having width comparable to a typical experimental resolution $\Delta \omega=10^{-2} 2 \pi \mathrm{c} / \mathrm{a}$ and averaged over photon polarizations) is shown in Fig. 3 . Bands are shown as a function of $\mathrm{k}_{\mathrm{z}}$ for three different values of $\theta$. We focus our description on the $\mathrm{L}-\mathrm{W}$ direction $\left(\phi=0^{\circ}\right)$, but examination of the results for other orientations leads to similar conclusions. Let us analyze first the results for $\theta=$ $0^{\circ}$. In this case the solution of Eq. (1)-(3) is very simple because $\mathrm{k}_{\|}=0$, and it yields the photonic bands along the $\Gamma-\mathrm{L}$ direction. The lowest energy gap shown in Fig. 3a around $\omega \mathrm{a} /(2 \pi \mathrm{c}) \sim 0.6$ appears clearly in the reduced DOS at the same values of the energy. It arises from diffraction in the backward direction by planes of the (111) family. The photonic bands at energies above the gap correspond to a photon momentum k outside the first Brillouin zone. Bands are then folded into the first BZ by subtracting from $\mathbf{k}$ a reciprocal lattice vector $(2 \pi / \mathrm{a})(1,1,1)$. These folded bands give rise to a finite DOS above the band gap.

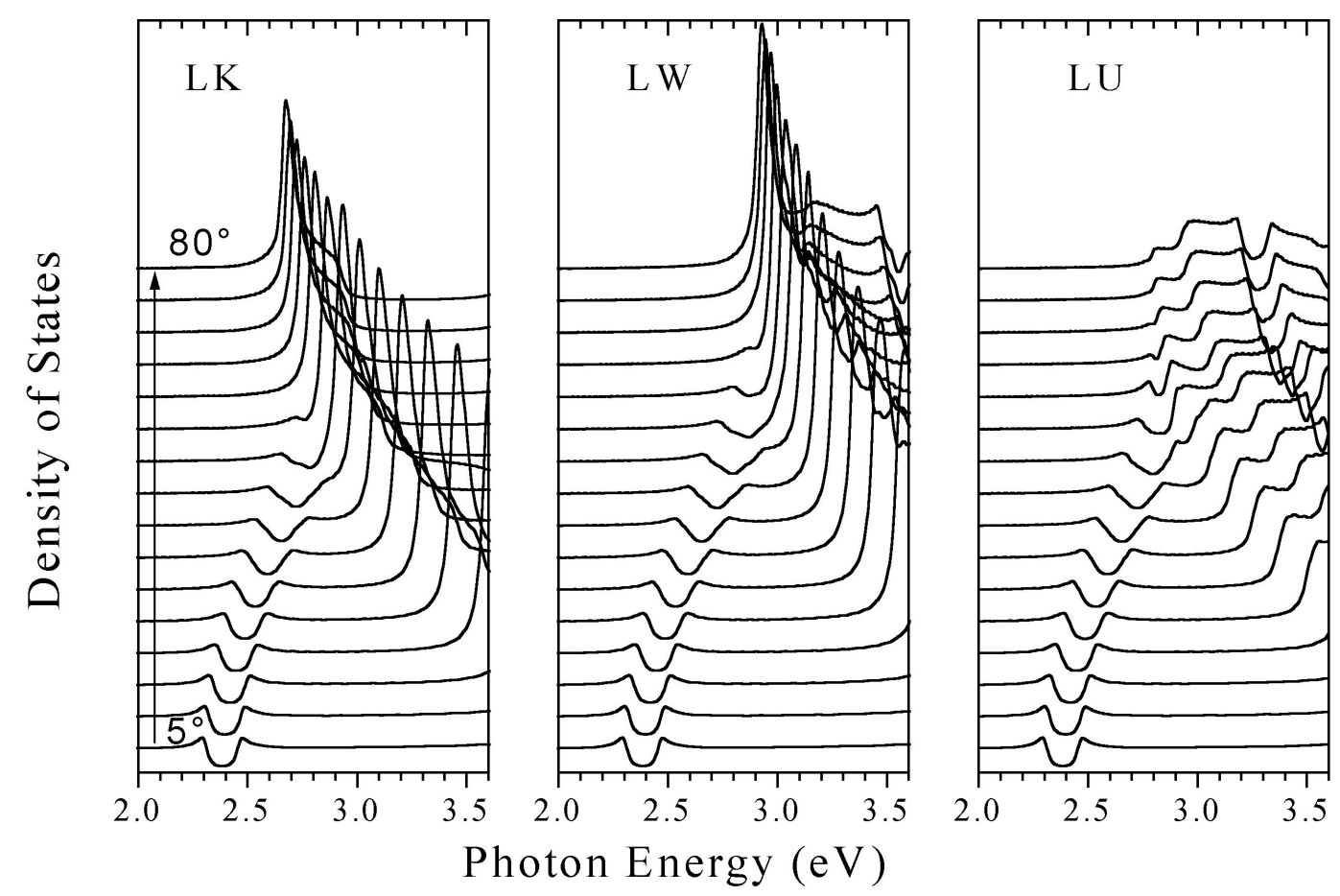

Fig. 4. Reduced density of states along the L-K, L-W and L-U crystallographic directions for incidence angles ranging from 5 to $80^{\circ}$ step by $5^{\circ}$. The different dispersion relation of band-gap and of van-Hove singularities is shown. The latter occurs at different frequencies for the different directions.

The reduced DOS reflects, of course, the energy dispersion of the photonic bands along the propagation direction. The latter is almost linear at low frequencies, and thus gives rise to a nearly constant one-dimensional DOS. For $\theta=0^{\circ}$, the second band just above the gap at L exhibits also an almost linear dispersion, thus the corresponding DOS in Fig. 3 remains above the gap. However, in analogy with electronic band structure of semiconductors, folded bands may exhibit a non-linear dispersion and can also show critical points (maxima, minima, or saddle points). In three dimensions, a critical point results in a divergence of the slope of the DOS (van Hove singularity). In the present onedimensional case the DOS itself diverges at a van Hove singularity. Notice that this phenomenon can occur at any angle of incidence, even for $\theta=0^{\circ}$. Anyway, it is well visible in Fig. 3 for larger values of $\theta$. When $\theta$ increases, the gap moves to higher energy, while the flat bands and the associated van Hove singularity move downwards to lower energy. Finally the gap and the flat bands overlap for $\theta \sim 70^{\circ}$. These features are finely reflected in the DOS, as 
reported in the right panels of Fig. 3. Thus the analysis of the reduced DOS and bands along the direction of propagation leads to a clear distinction between diffraction effects (sharp peaks in the DOS) from gaps or pseudogaps (deeps in the DOS). Critical points may give rise to van Hove singularities, which strongly enhance the DOS in the associated spectral region. The presence of a van Hove singularity marks the excitation of a photonic mode in the crystal and corresponds to diffraction in directions other than that of propagation.

Photonic modes give rise to several other features in the reduced DOS. All these features are associated with folded bands and exhibit a similar behavior as a function of $\theta$. In Fig. 4 we report the reduced DOS calculated for three different values of $\phi$ corresponding to the direction $\mathrm{L}-\mathrm{W}\left(\phi=0^{\circ}\right), \mathrm{L}-\mathrm{K}\left(\phi=-30^{\circ}\right)$ and $\mathrm{L}-\mathrm{U}\left(\phi=30^{\circ}\right)$. Notice that the energy scale has been determined for an opal with spheres of $222 \mathrm{~nm}$ of diameter, like those used for experiments. While for L-K and L-W directions similar reduced DOS are calculated, a remarkably different behavior is observed for the L-U direction. In this case the van Hove singularity remains very high in energy for any value of $\theta$. Steps seen in the DOS around $3 \mathrm{eV}$ are instead associated with bands having an almost linear dispersion. Each step indicates that a new band is becoming accessible. Furthermore, a second photonic gap appears for higher energies. Notice that this gap is also present for $\phi=0^{\circ}(\mathrm{L}-\mathrm{W})$, but is lacking for $\phi=30^{\circ}(\mathrm{L}-\mathrm{K})$.

Let us now analyze in detail the dependence on the angle $\phi$ (directions L-W, L-U and L-K). The low energy gap and its width have a weak dependence on $\phi$, while the spectral position of the Van-Hove singularity strongly depends on $\phi$. In particular, the peak of the reduced DOS for L-K and L-W directions are separated by about $0.2-0.3 \mathrm{eV}$, while for $\phi=$ $30^{\circ}$ (L-U direction) the van-Hove singularity remains almost outside the energy window.

It is evident that the energy position of the spectral features also depends on $\theta$. Pseudo-photonic gap and van Hove singularities have opposite dispersion on $\theta$. The stop band moves to higher energies upon $\theta$ increases while peaks associated with the van-Hove singularity move towards low energies. This is shown clearly in Figs. 4 for $\Gamma-\mathrm{K}$ and $\Gamma-\mathrm{W}$ directions. Notice also that the singularity peak merges with the photonic pseudo-gap around $\theta=70^{\circ}$.

\section{RESULTS AND DISCUSSION}

Figure $5 \mathrm{a}$ and $5 \mathrm{~b}$ show the variable-angle micro reflectance spectra of high quality opal for $\theta$ ranging from $5^{\circ}$ to $80^{\circ}$ in steps of $5^{\circ}$ with plane of incidence oriented along L-K and L-W directions, respectively. These spectra are vertically offset for a more clear view. At near-normal incidence a single, intense (reflectance almost identical to that of the Ag reference mirror), asymmetrical band associated with the stop band is observed at $2.47 \mathrm{eV}$ with a FWHM of about 0.15 $\mathrm{eV}$. When the incidence angle is increased from normal incidence up to $45^{\circ}$, the reflectance maximum shifts towards higher energies slightly reducing its intensity and modifying its shape. This behavior is accounted for by the theoretical band edge dispersion along the $\Gamma$-L direction. The agreement between calculated and measured gap position as well as its dependence on $\theta$ is very satisfactory. A more complex situation occurs for higher values of the incidence angle. We notice that for $\theta>50^{\circ}$ the shape of the spectrum is substantially changed and additional features occur. A structured band is observed to regularly shift its maximum in the range from 3 to $3.15 \mathrm{eV}$ retaining the dispersion of the stop band. It may roughly be assigned to the interplay of the stop band and of the excitation of the photonic modes observed in the DOS, which takes place for large $\theta$. The details of these features depend on the probed direction. However we also notice the appearance of weak features for the largest $\theta$ in the low-energy side of these bands. In particular, for L$\mathrm{W}$ direction the weak feature is at about $2.9 \mathrm{eV}$, while for the $\mathrm{L}-\mathrm{K}$ one is at about $2.7 \mathrm{eV}$. When $\theta$ decreases, these structures shift towards higher energies, i.e., they behave like the calculated van Hove singularity. Spectral features showing similar dispersions have been also previously observed in the transmission spectra of polystyrene [10] and silica [7,11] opal films. For $\theta$ of about $60^{\circ}$, these features cross the photonic stop band. Anyway, we notice that other weak spectral features with a negative dispersion as a function of the incidence angle may also be recognized in the range $20^{\circ}-60^{\circ}$ above the band gap peak (Fig. 5a and $5 \mathrm{~b}$ ). To give a better idea of the complicated structure of the spectra recorded for high energies and in order to find evidence for photonic modes, we enlarge (x10) the intensity scale to show some experimental curves in the right part of the figure. The behavior of such features with the incidence angle seems to continue the band dispersion of the former peaks observed for the largest angles (at lower energies). The energy of theoretical peaks of the reduced DOS (Fig. 4) are also reported as diamonds in Fig. 5a and 5b to make a direct comparison with the experimental spectra for each value of $\theta$. We found a very good agreement between the calculated peaks and the weak structures observed in the experimental spectra for both L-W and L-K directions. We therefore assign these features, having dispersion opposite to that of the stop band, to van Hove singularities in the reduced DOS, i.e., to diffraction from family planes other than the (111) one. 

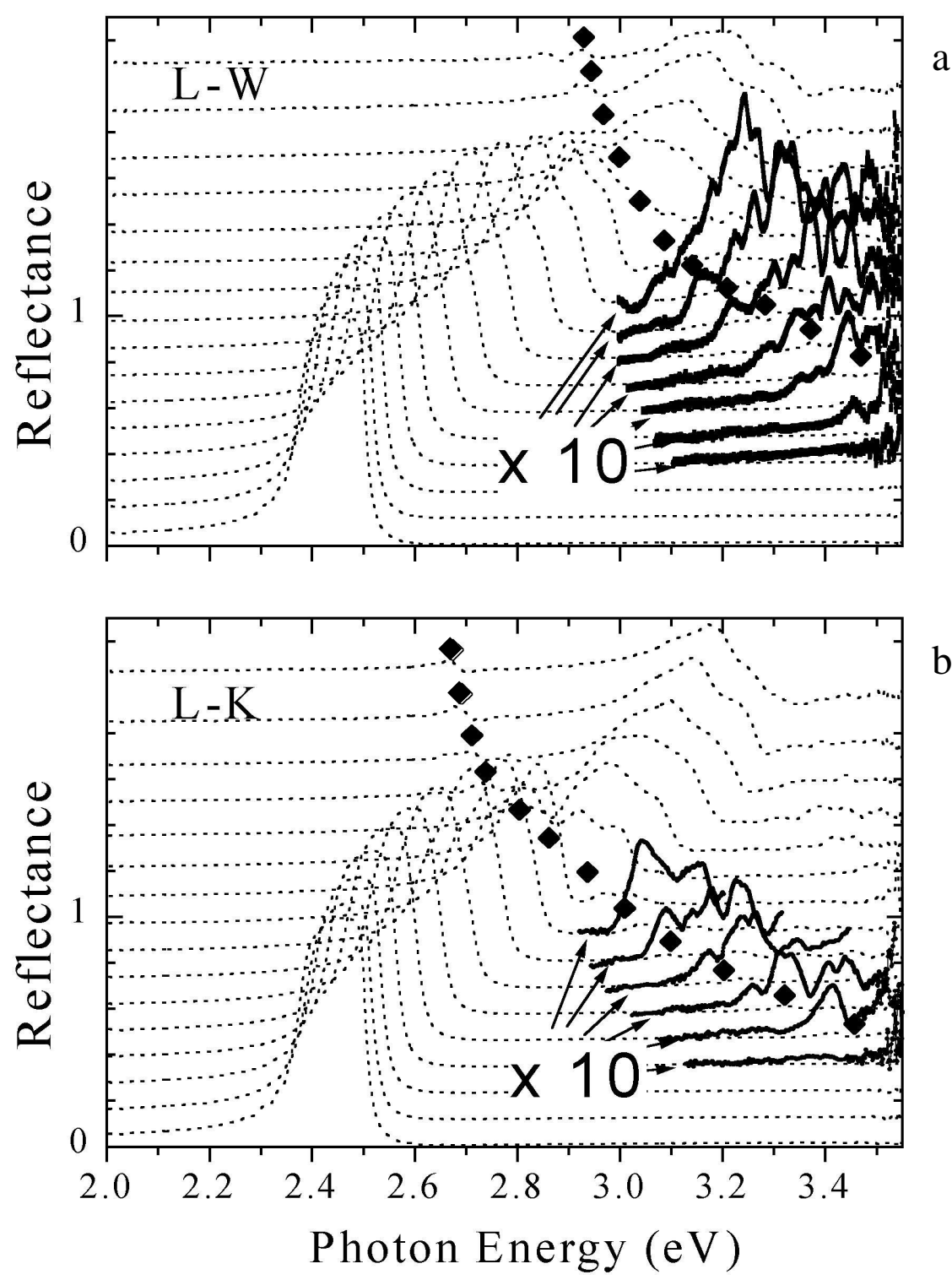

Fig. 5. Variable incidence angle Reflectance spectra of a $222 \mathrm{~nm}$ top quality opal. Spectra are recorded by steps of $5^{\circ}$ from 5 to 80 degrees. Upper (lower) panel: plane of incidence oriented along the L-W (L-K) direction. The high energy part of spectra corresponding to incidence angle from $20^{\circ}$ to $45^{\circ}\left(50^{\circ}\right)$ - indicated by arrows - have been multiplied by a factor 10 in order to show the very weak structures. Diamonds indicate the energy of the van-Hove singularities as calculated from theory (peaks in Figure 4) and have been placed for the same angles of incidence, close to the corresponding experimental curves.

Additional weak features exhibiting some dispersion can be also identified in the high energy part of the spectra for the largest angles. Their assignment is at present unknown.

In Fig. 6 we report the peak position and full width half maximum at $\theta=5^{\circ}$ as well as the van Hove singularities detected at $\theta=60,65$ and $70^{\circ}$ for opals grown with spheres with different diameters $(222,260,300,426 \mathrm{~nm})$. For the 222-nm opals data for L-W and L-K directions are reported. For other samples, measurements were performed with random orientation of the incidence plane. The stop band peak for $\theta=5^{\circ}$ scales with the inverse sphere diameters as expected. When straight lines with the same slope are reported starting for the van Hove singularities detected in the 222-nm opals for both L-W and L-K directions, all spectral features observed in different opals overlap one of these lines. These data are then indicative that van Hove singularities also scale as the main stop band thus confirming their 
assignment to an intrinsic property of the photonic band structure. Moreover, since refractive index of polystyrene is not dispersive in the spectral range here investigated, we do not expect significant influence on the data here discussed.

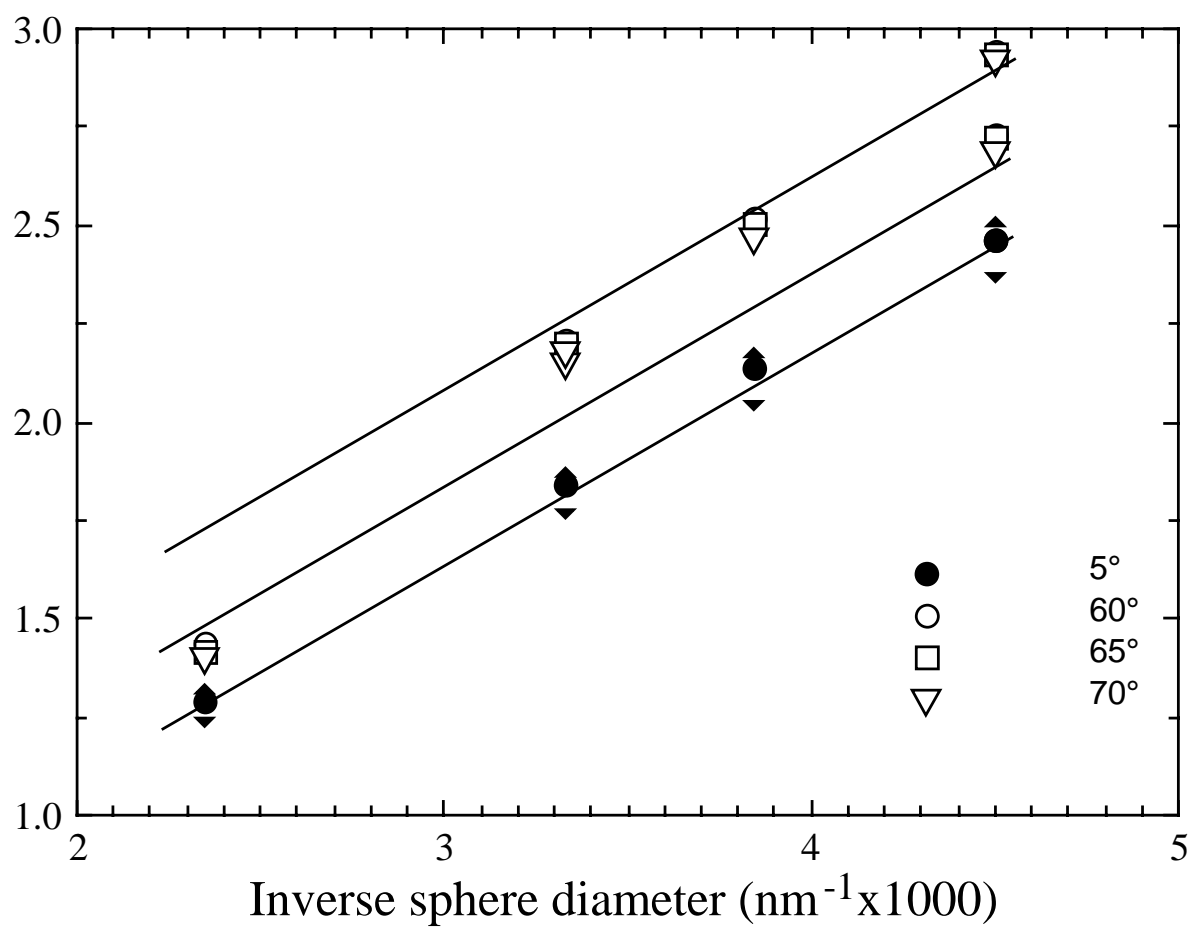

Fig. 6. Dependence on the inverse sphere diameter of spectral features in the variable incidence angle reflectance spectra. Full circle: energy peak of the stop band recorded at $\theta=5^{\circ}$; double arrow: full width half maximum of the stop band recorded at $\theta=5^{\circ}$; open circles, squares and triangules: spectral position of the features corresponding to van Hove singularities recorded at $\theta=60,65$ and 70 degrees, respectively. Full lines are guides for eyes parallel each other.

Measurements with polarized light both parallel and perpendicular to the incidence plane have been also performed on $222 \mathrm{~nm}$ opals. Even though these spectra have different shape, polarization does not seem to affect the energy position of the features assigned to the van Hove singularities. This is reasonable because the contribution of several different photonic modes to the singularities probably reduces the precise determination of their polarization. Investigations on this subject are still in progress.

\section{CONCLUSIONS}

A detailed study of the optical properties and photonic band structure for bulk artificial opals with close packed fcc lattice is reported. The reduced photonic bands and density of states corresponding to a given value of the incidence angle (or propagation direction) are calculated in order to provide a guide for the interpretation of optical spectra. Two main features with opposite dispersions have been identified. A minimum of the reduced DOS corresponds to the stop band in the propagation direction, while maxima and van Hove singularities in the reduced DOS are associated with the excitation of photonic modes in the crystal due to diffraction by higher order planes in the photonic lattice. These modes depend on the investigated orientations of the sample with respect to the incidence plane, reflecting the microscopic symmetries of the photonic lattice. There is a very good agreement between theoretical and experimental data for what concerns the dispersion of the stop band and van Hove singularities along L-W and L-K directions. The role of light polarization on the optical properties of opaline photonic crystals is currently under investigation.

\section{Acknowledgments}

We acknowledge support from the Ministry of the University and Scientific and Technological Research thorough the Fondo per gli Investimenti della Ricerca di Base (FIRB 2001-2003). 


\section{REFERENCES}

1 See for instance W.L. Vos, R. Sprik, A. van Bladeren, A. Imhof, A. Lagendijk, and G.H. Wegdam, Phys. Rev. B 53, 16231 (1996). Yu.A. Vlasov, V.N. Astratov, O.Z. Karimov, A.A. Kaplyanskii, V.N. Bogomolov, and A.V. Prokofiev, Phys. Rev. B 55, R13357 (1997). V.N. Bogomolov, S.V. Gaponenko, I.N. Germanenko, A.M. Kapitonov, E.P. Petrov, N.V. Gaponenko, A.V. Prokofiev, A.N. Ponyanina, N.I. Silvanovich, and S.M. Samoilovich, Phys. Rev. E 55, 7619 (1997). H. Miguez, C. Lopez, F. Meseguer, A. Blanco, L. Vazquez, R. Mayoral, M. Ocana, V. Fornes, and A. Mifsud, Appl. Phys. Lett. 71, 1148 (1997). H. Miguez, A. Blanco, F. Meseguer, C. Lopez, H.M. Yates, M.E. Pemble, V. Fornes, and A. Mifsud, Phys. Rev. B 59, 1563 (1999). M. Thijssen, R. Sprik, J.E.G.J. Wijnhoven, M. Megens, T. Narayanan, A. Lagendijk, and W.L. Vos, Phys. Rev. Lett. 83, 2730 (1999). Yu. A. Vlasov, V.N. Astratov, A.V. Baryshev, A.A. Kaplyanskii, O.Z. Karimov, and M.F. Limonov, Phys. Rev. E 61, 5784 (2000). H.P. Schriemer, H.M. van Driel, A.F. Koenderink, and W.L. Vos, Phys. Rev. A 63, 011801 (2000).). A.F. Koenderink, L. Bechger, H.P. Schriemer, A. Lagendijk, and W.L. Vos, Phys. Rev. Lett. 88, 143903 (2002). J.F.G. Lopez and W.L. Vos, Phys. Rev. E 66, 036616 (2002). V.N. Astratov, A.M. Adawi, S. Fricker, M.S. Skolnick, D.M. Whittaker, and P.N. Pusey, Phys. Rev. B 66, 165215 (2002).

2 I.I. Tarhan and G.H. Watson, Phys. Rev. Lett. 76, 315 (1996).

3 H.M. van Driel and W.L. Vos, Phys. Rev. B 62, 9872 (2000). W.L. Vos and H.M. van Driel, Phys. Lett. A 272,101 (2000).

4 S.G. Romanov, T. Maka, C.M. Sotomayor Torres, M. Muller, R. Zentel, D. Cassagne, J. Manzanares-Martinez, and C. Jouanin, Phys. Rev. E 63, 056603 (2001

5 H.S. Sozuer, J.W. Haus, and N. Inguva, Phys. Rev. B 45 (1992) 13962. K. Busch, and S. John, Phys. Rev. E 58 (1998) 3896. A. Reynolds, F. Lopez-Tejeira, D. Cassagne, F.J. Garcia-Vidal, C. Jouanin, and J. Sanchez-Dehesa, Phys. Rev. B60, 11422 (1999). V. Yannopapas, N. Stefanou, and A. Modinos, J. Phys: Cond. Matt. 9, 10261 (1997). Z.-Y. Li, and Z.-Q. Zhang, Phys. Rev. B 62, 1516 (2000). F. Lopez-Tejeira, T. Ochiai, K. Sakoda, and J. Sanchez-Dehesa, Phys. Rev. B 65, 195110 (2002). Z.L. Wang, C.T. Chan, W.Y. Zhang, Z. Chen, N.B. Ming, and P. Sheng, Phys. Rev. E 67, 016612 (2003).

6 V. Yannopapas, N. Stefanou, and A. Modinos, Phys. Rev. Lett. 86, 4811 (2001).

7 H. Miguez, V. Kitaev, and G.A. Ozin, Appl. Phys. Lett. 84, 1239 (2004).

8 J.F. Galisteo-Lopez, E. Palacios-Lidon, E. Castillo-Martinez and C. Lopez, Phys. Rev. B68, 115109 (2003).

9 E. Pavarini, L.C. Andreani, C. Soci, M. Galli, F. Marabelli, and D. Comoretto, Phys. Rev. B, submitted

10 D. Comoretto, R. Grassi, F. Marabelli, and L.C. Andreani, Mat. Sci Eng. C 23, 61 (2003); D. Comoretto, F. Marabelli, C. Soci, M. Galli, E. Pavarini, M. Patrini and L.C. Andreani, Synthetic Met. 139, 633 (2003).

11 D. Comoretto, D. Cavallo, G. Dellepiane, R. Grassi, F. Marabelli, L.C. Andreani, C.J. Brabec, A. Andreev, and A.A. Zakhidov, Mat. Res. Soc. Symp. Proc. 708, BB10.19.1 (2002).

12 A similar behaviour has been also previously reported in ref. 8 and attributed to the coincidence of the energy bands for the L-U and L-K direction in the Brollouin zone.

13 K.M. Ho, C.T. Chan, and C.M. Soukoulis, Phys. Rev. Lett. 65, (1990) 3152.

14 We adopt here standard reduced units where $c$ is the speed of light, and $a=d \sqrt{ } 2$ is the lattice spacing for close packed condition. 